\title{
Analysis of the color patterns of early gastric cancer using an autofluorescence imaging video endoscopy system
}

\author{
Motohiko Kato ${ }^{1,2}$, Noriya Uedo ${ }^{2,3}$, Ryu Ishihara $^{2}$, Takashi Kizu ${ }^{1}$, Rika Chatani ${ }^{2}$, Takuya Inoue ${ }^{2}$, \\ Eriko Masuda $^{5}$, Koichi Tatsumi ${ }^{6}$, Yoji Takeuchi ${ }^{2}$, Koji Higashino ${ }^{2}$, Hiroyasu Iishi $^{2}$, Yasuhiko Tomita $^{4}$, \\ and Masaharu TAtsuta ${ }^{3}$ \\ ${ }^{1}$ Department of Gastroenterology and Hepatology, Osaka University Graduate School of Medicine, Osaka, Japan \\ ${ }^{2}$ Department of Gastrointestinal Oncology, Osaka Medical Center for Cancer and Cardiovascular Diseases, 1-3-3 Nakamichi, Higashinari-ku, \\ Osaka 537-8511, Japan \\ ${ }^{3}$ Department of Endoscopic Training and Learning Center, Osaka Medical Center for Cancer and Cardiovascular Diseases, Osaka, Japan \\ ${ }^{4}$ Department of Pathology, Osaka Medical Center for Cancer and Cardiovascular Diseases, Osaka, Japan \\ ${ }^{5}$ Department of Internal Medicine, Hyogo Prefectural Nishinomiya Hospital, Nishinomiya, Japan \\ ${ }^{6}$ Department of Internal Medicine, Dongo Hospital, Yamatotakada, Nara, Japan
}

\begin{abstract}
Background. Using a novel autofluorescence imaging video endoscopy system (AFI), tumors in the esophagus and the colon appeared purple in a green background, but the color patterns of early gastric cancer (EGC) were found to vary. Factors associated with these patterns remain unknown. The aims of the present study were to classify the color patterns of EGCs and to investigate the correlation between the patterns and clinicopathological features.

Methods. A total of 107 EGCs that had been evaluated by AFI endoscopy, prior to endoscopic or surgical resection, were included. The color patterns of EGCs in AFI images and the association between tumor color and clinicopathological factors were evaluated. These factors included tumor morphology, location, size, background color, histological type, depth of invasion, lymphatic or vessel permeation, and ulceration.

Results. The color patterns of EGCs were classified into the following four groups: purple tumors in a green background $(52 \%)$; green tumors with a purple margin in a green background (21\%); green tumors in a purple background $(17 \%)$; and purple tumors in a purple background $(10 \%)$. Univariate analysis showed that macroscopic type, histological type, ulceration, and background AFI color were significantly associated with tumor color, whereas multivariate analysis revealed that macroscopic type was the only independent contributor to tumor color.

Conclusion. The present study has enabled a clearer understanding of the significance of tumor color in relation to the AFI imaging of EGCs. Recognition of the color patterns in AFI images should help in the diagnosis of EGCs.
\end{abstract}

Key words Autofluorescence endoscopy - Early gastric cancer - Atrophic gastritis · Tumor color patterns

Offprint requests to: N. Uedo

Received: September 27, 2009 / Accepted: October 27, 2009

\section{Introduction}

Early diagnosis and treatment can improve the prognosis of gastric cancers. Despite the progressive development of endoscopic modalities [1], the early detection of superficial neoplasms during routine esophagogastro-duodenoscopy (EGD) remains difficult because there are few morphological changes that differentiate malignant from nonmalignant lesions. Moreover, although treatments such as endoscopic mucosal resection [2] or endoscopic submucosal dissection (ESD) [3] are widely performed for the local resection of EGCs in Japan, accurate diagnosis of tumor extent is sometimes difficult because EGCs occasionally have flat or isochromatic tumor extensions. Chromoendoscopy can increase diagnostic yields in relation to the detection and delineation of flat tumors, by the enhancement of morphological features [4]. However, this modality is not widely used in clinical practice because its deployment can result in substantial prolongation of routine endoscopic examinations. Consequently, easier and more efficacious endoscopic modalities for diagnosing EGC are needed.

An autofluorescence imaging videoendoscopy (AFI) system produces real-time pseudocolor images from the computed detection of autofluorescence emitted by endogenous fluorophores (collagen, nicotinamide, adenine dinucleotide, flavin, and porphyrins) due to excitation by light. The system can identify lesions, including malignancies, by detecting differences in tissue fluorescence properties, and can thus reveal earlystage cancers that are not detectable by conventional white-light endoscopy [2].

In a previous study, when we investigated the diagnostic ability of an AFI system for early-stage cancers in the digestive tract, we discovered that tumors in the 
esophagus and the colon appeared purple in a green background [5]. However, the color pattern of EGCs in the AFI images varied among tumors. The factors associated with these color variations were not investigated at that time [5]. The aims of the present study were to evaluate the endoscopic appearance of EGCs in AFI images and to investigate the clinicopathological factors associated with different tumor colors.

\section{Patients and methods}

\section{Study sample}

Since September 2003, the data of patients who have visited our endoscopy unit at Osaka Medical Center for Cancer and Cardiovascular Diseases, and who have undergone AFI, have been recorded consecutively in a database that is maintained prospectively and regularly updated. The input clinicopathological data were compiled according to the Japanese classification of gastric carcinoma protocol [6]. From this database, patients with EGC who presented between June 2004 and January 2006 were retrieved and their main tumors were included in the study. Patients with a history of gastrectomy were excluded. If a patient had multiple lesions, the largest one was selected for analysis. Approval from the Institutional Review Board at our medical center was obtained for this study.

A total of 127 consecutive patients with EGC who underwent AFI for pretherapeutic evaluation were identified from the database. Seven patients with a history of gastrectomy and one patient who transferred to another hospital were excluded. Among the 119 EGC lesions in the 119 patients involved in the study, AFI images were insufficient for evaluation in 10 lesions, and 2 lesions could not be classified. Therefore, a final total of 107 lesions were analyzed in this study.

\section{Endoscopic procedure}

The AFI system used in this study consisted of a light source (CLV-260SL; Olympus Medical Systems, Tokyo, Japan), a processor (CV-260SL; Olympus), a video monitor, and a video endoscope (EVIS-FQ260Z; Olympus) that was equipped with two charged-coupled devices (CCDs) that were available with autofluorescence and white-light modes. In the autofluorescence mode, blue excitation light $(395-475 \mathrm{~nm})$ to induce autofluorescence and green light $(540-560 \mathrm{~nm})$ to capture green reflection images were sequentially emitted from the light source through a rotation filter. A cut filter that was placed with the lens was used to permit only light with wavelengths between 490 and $625 \mathrm{~nm}$ to intensify the CCD for the AFI mode [7]. All examinations were performed by a single endoscopist (N.U.) who had 4 years' experience with autofluorescence endoscopy and 14 years' experience with conventional endoscopy.

Five minutes before the examination, patients ingested a mixture of a mucolytic agent, $20000 \mathrm{U}$ pronase (Pronase MS; Kaken Pharmaceutical, Tokyo, Japan), a defoaming agent, $80 \mathrm{mg}$ dimethylpolysiloxane syrup (Gascon Drops; Kissei Pharmaceutical, Matsumoto, Japan), and $1 \mathrm{~g}$ sodium bicarbonate diluted in $100 \mathrm{ml}$ of tap water. After the application of topical anesthesia, the endoscope was gently inserted into the stomach. First, the color of the background mucosa and tumors were evaluated under AFI observation, and at least four AFI images of each tumor were taken from various viewing angles. After that, the tumors were thoroughly investigated by conventional white-light endoscopy. This was followed by $0.04 \%$ indigo carmine chromoendoscopy. Images obtained from the whitelight endoscopy and chromoendoscopy were recorded. All images were digitally stored on an image server (Solemio Endo; Olympus).

\section{Analysis of color patterns of EGC}

Two endoscopists (M.K. and N.U.) reviewed the recorded AFI images, and the color patterns were classified into the following four types on the basis of tumor and background color: (1) a purple tumor on a green background (P/G type); (2) a green tumor on a green background (G/G type); (3) a green tumor on a purple background (G/P type); and (4) a purple tumor on a purple background (P/P type). When a tumor was located on a background color border, the color which surrounded more than half of the circumference of the tumor was designated as a background color.

The association between tumor color in the AFI images and a range of clinicopathological factors was investigated. These factors included: tumor size $(\leq 2 \mathrm{~cm}$ or $>2 \mathrm{~cm}$ ), location (upper, middle, or lower third), macroscopic type (elevated or depressed), histological type (differentiated or undifferentiated), and depth of invasion (mucosal or submucosal); the presence or absence of vessel invasion; and background AFI color. For the factors that had a significant association on univariate analysis, multivariate analysis was performed to assess the strength and independence of the association. The macroscopic type of the tumor was determined under chromoendoscopic observation. Types 0I, OIIa, and OIIa+IIc were classified as elevated type. Types 0IIc and OIIc+IIa were classified as depressed type. Type 0IIb (flat) and type 0III (excavated) were not found in the present study sample. 


\section{Statistical analysis}

Stat View version 5.0 (SAS Institute, Cary, NC, USA) was used for data analysis. The $\chi^{2}$ test and Fisher's exact probability test, when appropriate, were used for univariate analysis of the association between tumor AFI color and clinicopathological factors. Logistic regression analysis was performed for multivariate analysis. A $P$ value of less than 0.05 was considered to be statistically significant.

\section{Results}

\section{AFI color patterns of early gastric cancers}

The characteristics of the EGCs are detailed in Table 1. The distribution of the color patterns of the EGCs observed in AFI images is shown in Fig. 1. The P/G- and G/P-type tumors could be easily identified due to clear differences in color (Figs. 2 and 3). For G/G type tumors, both the tumor and the background mucosa color were

Table 1. Clinical characteristics of the study subjects

\begin{tabular}{ll}
\hline Number of patients & 119 \\
Mean age (years) & $70(9)^{\mathrm{a}}$ \\
Sex (\%) & Men: 77 \\
& Women: 23 \\
Treatment (\%) & Endoscopy: 86 \\
& Surgery: 14 \\
Location (\%) & U: 29 \\
& M: 44 \\
& L: 27 \\
Mean tumor size (mm) & $21.4(15.0)$ \\
Macroscopic type (\%) & OI: 3 \\
& OIIa: 42 \\
& OIIa+IIc: 3 \\
& OIIc+IIa: 3 \\
Histological type (\%) & OIIc: 49 \\
& Pap: 3 \\
Tub1: 73 \\
Tub2: 16 \\
Por: 4 \\
Depth of invasion (\%) & Sig: 4 \\
& Mucosal: 79 \\
Vessel invasion (\%) & Submucosal: 21 \\
Ulceration (\%) & Absent: 91 \\
& Present: 9 \\
Tumor AFI color (\%) & Absent: 87 \\
& Present: 13 \\
& Purple: 56 \\
& Green: 34 \\
& Not evaluable: 10 \\
& Purple: 28 \\
& Green: 72 \\
\end{tabular}

AFI, autofluorescence imaging videoendoscopy; U, upper third; M, middle third; L, lower third; Pap, papillary adenocarcinoma; Tub1, well-differentiated tubular adenocarcinoma; Tub2, moderately differentiated tubular adenocarcinoma; Por, poorly differentiated adenocarcinoma; Sig, signet-ring cell carcinoma

${ }^{a}$ Numbers in parentheses are SDs green. However, the tumors usually had a purple margin and could therefore be differentiated from the background mucosa (Fig. 4). For both G/P- and G/G-type tumors, purple nodules were sometimes seen inside the green tumors. P/P-type tumors exhibited a color similar to that of the background mucosa and could only be recognized by their shape.

\section{Factors associated with AFI tumor color}

Univariate analysis showed that macroscopic type, histological type, the presence of ulceration, and background AFI color were significantly associated with tumor color (Table 2). However, when multivariate analysis was used to further assess these factors, only macroscopic type was independently associated with tumor color (Table 3).

\section{Discussion}

In the present study, we found that EGCs in AFI images could be classified according to the tumor and background color, and that the factor most strongly associated with tumor color was the macroscopic type. In AFI images, almost all tumors with an elevated appearance (elevated type) were purple, while most of the tumors with a depressed appearance (depressed type) were green. Although endogenous fluorophores exist in both the mucosa and the submucosa, collagen in the submucosa discharges a strong green autofluorescence [8]. AFI images differ according to the autofluorescence properties of the tissue, and the intensity of light, in particular, affects the AFI color. Areas with strong autofluorescence appear bright green and areas with weak autofluorescence appear purple or greenish-

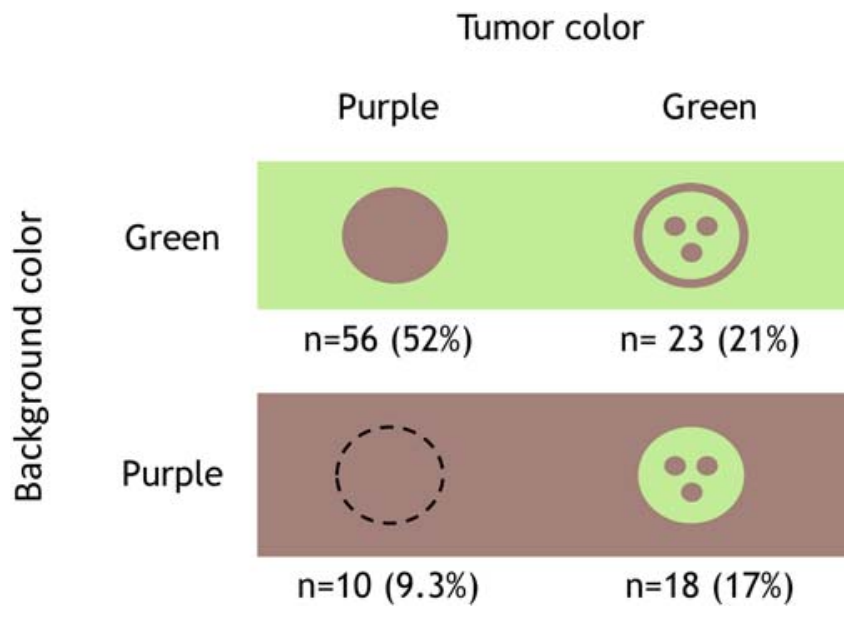

Fig. 1. Color patterns and prevalence of early gastric cancers in autofluorescence imaging videoendoscopy (AFI) 

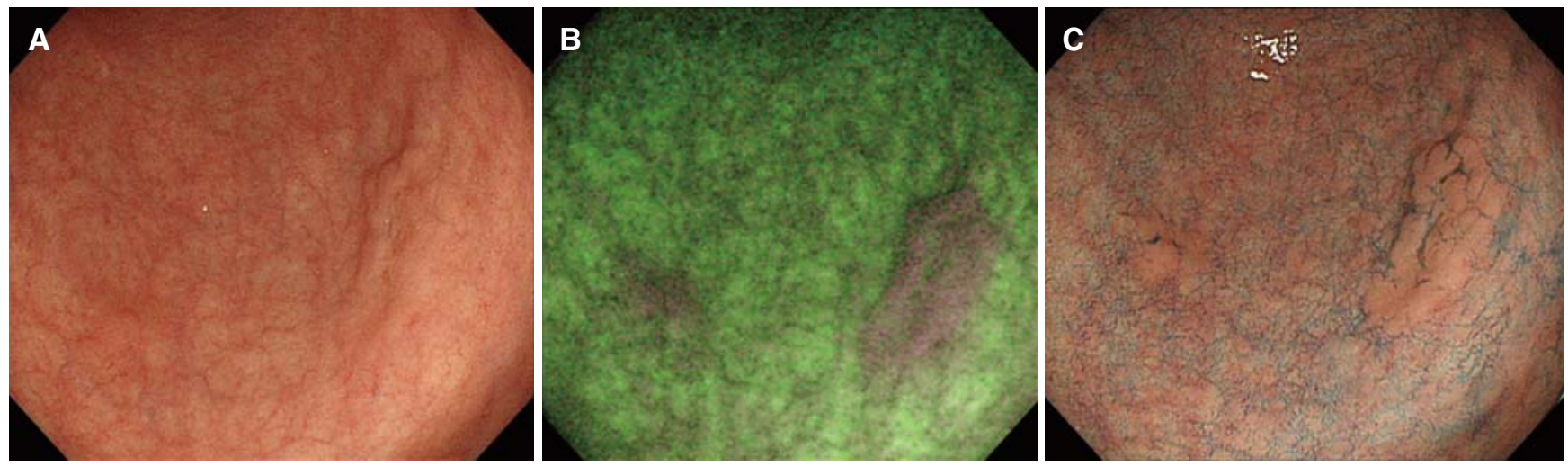

Fig. 2A-C. Endoscopic images of a purple tumor on a green background (P/G)-type tumors. A Conventional white-light image showing a slightly elevated tumor. However, its extent is unclear. B AFI image depicting the tumor as purple areas in a green background. C Image of two elevated tumors with contrasting topography visualized using chromoendoscopy with indigo carmine. The tumor was identified as a differentiated adenocarcinoma and removed endoscopically
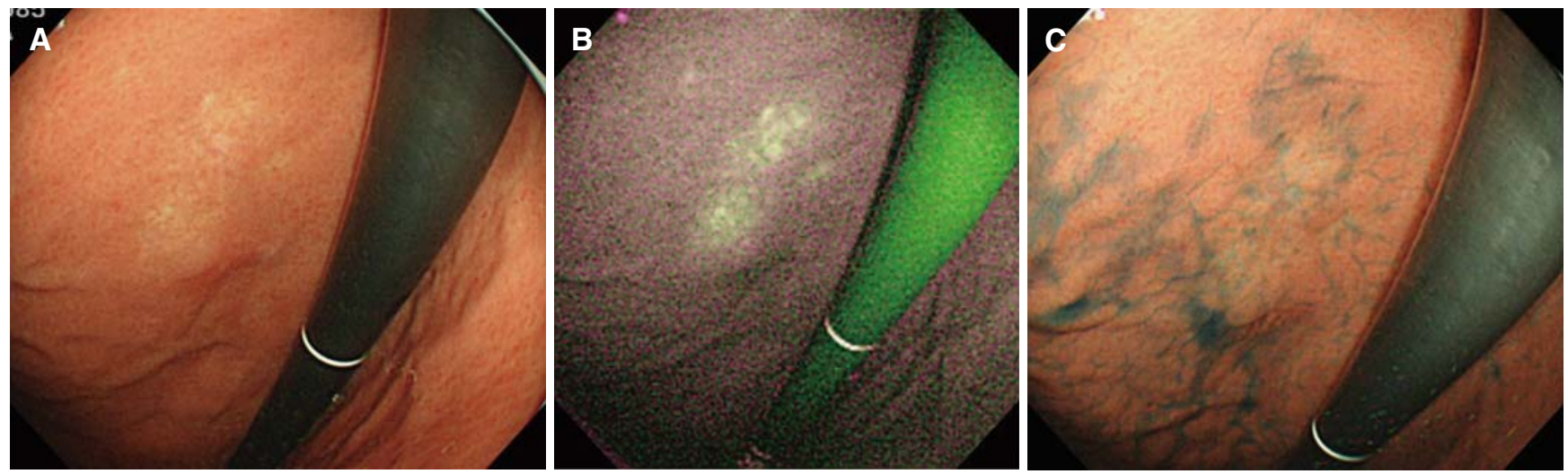

Fig. 3A-C. Endoscopic images of a green tumor on a purple background (G/P)-type tumor. A Image obtained using white-light endoscopy. Tumors appear as vague whitish areas. B AFI image showing the tumors as green areas in a purple background. C Image obtained using chromoendoscopy and showing a shallow depressed tumor located in the lower gastric body. An endoscopically resected specimen revealed undifferentiated adenocarcinoma confined to the mucosa
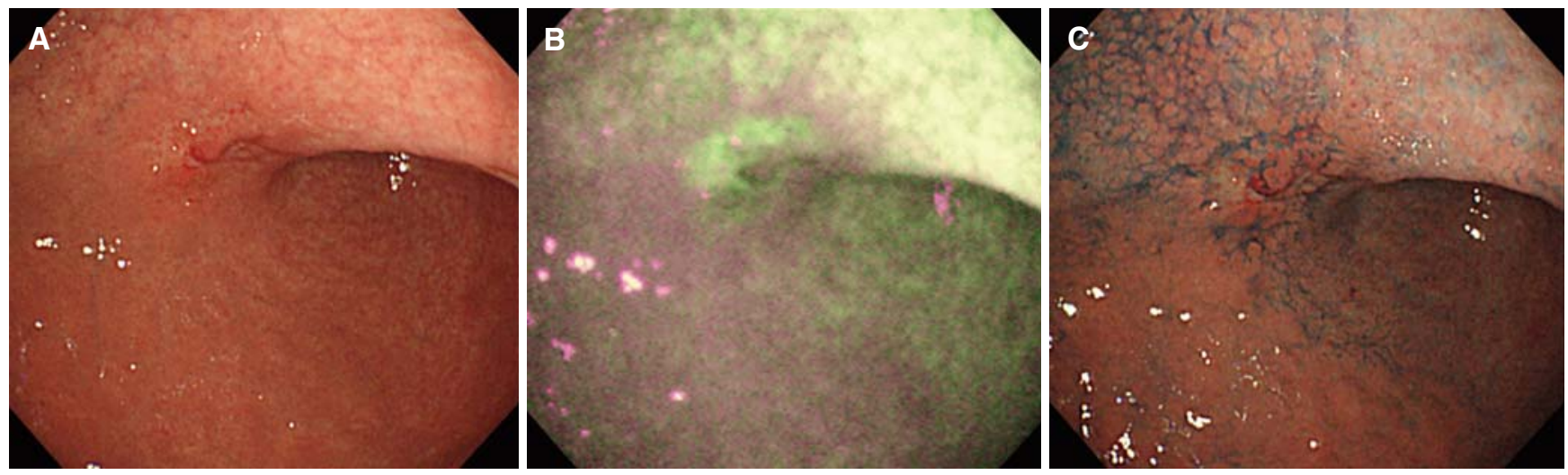

Fig. 4A-C. Endoscopic images of a green tumor on a green background (G/G)-type tumor. A Irregular reddish mucosa in the anterior wall of the lower gastric body. B AFI image. The tumor appears as a green area with a purple margin. A purple nodule is located in the center of the lesion. The tumor was located in an area adjacent to the purple-colored background mucosa, but was mostly surrounded by green background that indicated areas with chronic atrophic fundal gastritis. C Chromoendoscopic image revealing a depressed-type tumor with a central nodule. This tumor was identified histologically as a well-differentiated tubular adenocarcinoma 
Table 2. Univariate analysis of factors associated with the color of lesions in AFI images

\begin{tabular}{|c|c|c|c|}
\hline & $\begin{array}{l}\text { No. of green } \\
\text { tumors }\end{array}$ & $\begin{array}{l}\text { No. of purple } \\
\text { tumors }\end{array}$ & $P$ value \\
\hline \multicolumn{4}{|l|}{ Location } \\
\hline Upper third & 10 & 17 & \multirow[t]{3}{*}{0.088} \\
\hline Middle third & 24 & 26 & \\
\hline Lower third & 7 & 23 & \\
\hline \multicolumn{4}{|l|}{ Size } \\
\hline$<20 \mathrm{~mm}$ & 19 & 37 & \multirow[t]{2}{*}{0.328} \\
\hline$\geq 20 \mathrm{~mm}$ & 22 & 29 & \\
\hline \multicolumn{4}{|l|}{ Macroscopic type } \\
\hline Elevated & 4 & 51 & \multirow[t]{2}{*}{0.000} \\
\hline Depressed & 37 & 15 & \\
\hline \multicolumn{4}{|l|}{ Histological type } \\
\hline Differentiated & 33 & 65 & \multirow[t]{2}{*}{0.001} \\
\hline Undifferentiated & 8 & 1 & \\
\hline \multicolumn{4}{|l|}{ Depth of invasion } \\
\hline Mucosal & 36 & 48 & \multirow{2}{*}{0.065} \\
\hline Submucosal & 5 & 18 & \\
\hline \multicolumn{4}{|l|}{ Vessel invasion } \\
\hline Absent & 32 & 57 & \multirow[t]{2}{*}{0.147} \\
\hline Present & 9 & 9 & \\
\hline \multicolumn{4}{|l|}{ Ulceration } \\
\hline Absent & 32 & 62 & \multirow[t]{2}{*}{0.018} \\
\hline Present & 9 & 4 & \\
\hline \multicolumn{4}{|l|}{$\begin{array}{l}\text { Background color } \\
\text { in AFI image }\end{array}$} \\
\hline Green & 23 & 56 & \multirow[t]{2}{*}{0.001} \\
\hline Purple & 18 & 10 & \\
\hline
\end{tabular}

Table 3. Multivariate analysis of factors associated with green tumor color in AFI images

\begin{tabular}{|c|c|c|}
\hline & Odds ratio $(95 \% \mathrm{CI})$ & $P$ value \\
\hline \multicolumn{3}{|l|}{ Macroscopic type } \\
\hline Elevated & 1 & \\
\hline Depressed & $24.9(7.12-87.3)$ & 0.000 \\
\hline \multicolumn{3}{|l|}{ Background color } \\
\hline Green & 1 & \\
\hline Purple & $2.56(0.62-9.09)$ & 0.144 \\
\hline \multicolumn{3}{|l|}{ Histological type } \\
\hline Differentiated & 1 & \\
\hline Undifferentiated & $2.54(0.26-2.43)$ & 0.419 \\
\hline \multicolumn{3}{|l|}{ Ulceration } \\
\hline Absent & 1 & \\
\hline Present & $1.00(0.22-4.55)$ & 0.999 \\
\hline
\end{tabular}

CI, confidence interval

purple. Therefore, we speculate that the elevated-type tumor reduces autofluorescence from the submucosa and thus appears purple in AFI images, and that most of the depressed-type tumors do not affect autofluorescence intensity because they are thin and therefore appear green. In contrast to colon or esophageal tumors, most EGCs have been found to be of a depressed macroscopic type [9]. Therefore, their color would be green, which is uncommon in other regions of the digestive tract.
Histological type and background AFI color were two factors that were found to be significantly associated with tumor color on univariate analysis. However, this association did not prove to be the case on multivariate analysis. We believe that there are a number of reasons for this. With regard to morphology, although differentiated-type EGCs have the appearance of both elevated- and depressed-type tumors, undifferentiatedtype EGCs are mostly of the depressed type [10]. As for the background color, the color of the gastric body mucosa is closely related to the grade of atrophic fundal gastritis [11]. The normal fundic mucosa looks purple, whereas abnormal mucosa with gastritis appears green. Our chromoendoscopic investigation showed that undifferentiated-type EGCs were likely to develop in the areas adjacent to, or sometimes inside, the normal fundic mucosa [12], which appears purple in AFI images. The undifferentiated EGCs are likely to be of the depressed type and, therefore, look green. By contrast, differentiated-type EGCs that are often of the elevated type frequently look purple and develop in areas with atrophic fundal gastritis or in the pyloric mucosa [12]. The pyloric mucosa appears green in AFI images.

In $\mathrm{P} / \mathrm{G}$ - and G/P-type tumors, we found that the tumor profile was well delineated in the AFI images. We compared the diagnostic ability of AFI, white-light endoscopy, and chromoendoscopy for the extent of the EGC lesions. It was found that the accuracy of AFI was not as good as that of chromoendoscopy, but that it was better than white-light endoscopy [5]. Mucosal thickening or edema caused by ulceration and scarring looked purple, mimicking the tumor color. In some cases, this led to the misdiagnosis of tumor extent. Consequently, AFI may not be suitable for the evaluation of lesions with ulceration or scars. We believe, therefore, that chromoendoscopy is still necessary for pretreatment examination, although AFI would be a useful adjunct in routine EGD, because it does not require a troublesome dye spraying procedure and is less time-consuming.

Our study had several limitations. Patients with EGC who were referred for endoscopic resection accounted for more than $80 \%$ of our study subjects. Therefore, our study may not reflect the ECG profile in the actual population. In other words, the majority of the ECGs evaluated in our study were small, elevated, and differentiated types of mucosal EGCs. To correct for such bias in the analysis of factors associated with tumor color, we performed multivariate analysis. This revealed that the strongest independent correlation was between tumor color and macroscopic type.

The incidence of the P/P-type tumor was relatively low as compared with that of the other types of EGC tumor. For tumor types where the tumor color and background color differed, such as was the case with the 
$\mathrm{P} / \mathrm{G}$ and $\mathrm{G} / \mathrm{P}$ types, the tumor was clearly delineated. However, for tumors that had a color similar to that of the background mucosa, it was sometimes difficult to identify them by their color. Although G/G-type tumors were frequently associated with a purple rim or central nodules, and were recognized by their color, P/P-type tumors were the most difficult to recognize. As a consequence, it is possible that some of these tumors were missed in the screening process using AFI.

In conclusion, the present study has enabled a clearer understanding of the significance of tumor color in relation to the AFI imaging of EGCs. The color pattern of the EGCs was classified into four types and their color appeared to be primarily associated with the macroscopic type of the tumor. Recognition of these color patterns should facilitate a clearer interpretation of endoscopic findings in relation to AFI diagnosis.

\section{References}

1. The Paris endoscopic classification of superficial neoplastic lesions: esophagus, stomach, and colon: November 30 to December 1, 2002. Gastrointest Endosc 2003;58:S3-43.

2. Uedo N, Iishi H, Tatsuta M, Ishihara R, Higashino K, Takeuchi $\mathrm{Y}$, et al. Long term outcomes after endoscopic mucosal resection for early gastric cancer. Gastric Cancer 2006;9:88-92.

3. Takeuchi $\mathrm{Y}$, Uedo $\mathrm{N}$, Iishi $\mathrm{H}$, Yamamoto $\mathrm{S}$, Yamamoto $\mathrm{S}$, Yamada T, et al. Endoscopic submucosal dissection with insulated-tip knife for large mucosal early gastric cancer: a feasibility study (with videos). Gastrointest Endosc 2007;66:186-93.

4. Ohkawa A, Miwa H, Namihisa A, Kobayashi O, Nakaniwa N, Ohkusa T, et al. Diagnostic performance of light-induced fluorescence endoscopy for gastric neoplasms. Endoscopy 2004;36: 515-21.

5. Uedo N, Iishi H, Tatsuta M, Yamada T, Ogiyama H, Imanaka K, et al. A novel videoendoscopy system by using autofluorescence and reflectance imaging for diagnosis of esophagogastric cancers. Gastrointest Endosc 2005;62:521-8.

6. Japanese Gastric Cancer Association. Japanese classification of gastric carcinoma, 2nd English edition. Gastric Cancer 1998;1: $10-24$.

7. Uedo N, Iishi H, Ishinhara R, Higashino K, Takeuchi Y. Novel autofluorescence videoendoscopy imaging system for diagnosis of cancers in the digestive tract. Dig Endosc 2006;18(Suppl 1): S131-6.

8. Izuishi K, Tajiri H, Fujii T, Boku N, Ohtsu A, Ohnishi T, et al. The histological basis of detection of adenoma and cancer in the colon by autofluorescence endoscopic imaging. Endoscopy 1999; 31:511-6.

9. Everett SM, Axon AT. Early gastric cancer in Europe. Gut 1997; 41:142-50.

10. Fujishiro M, Oka M, Yahagi N, Nakamura M, Kakushima N, Kodashima S, et al. Correlation of serum pepsinogens and gross appearances combined with histology in early gastric cancer. J Exp Clin Cancer Res 2006;25:207-12.

11. Inoue $T$, Uedo N, Ishihara R, Kawaguchi T, Kawada N, Chatani $\mathrm{R}$, et al. Autofluorescence imaging videoendoscopy in the diagnosis of chronic atrophic fundal gastritis. J Gastroenterol 2009. doi:10.1007/s00535-009-0150-7.

12. Tatsuta M, Okuda S, Taniguchi H, Tamura H. Gross and histological types of early gastric carcinomas in relation to the acid secreting area. Cancer 1979;43:317-21. 